NASZA DERMATOLOGIA Online OUR DERMATOLOGY Online

Source of Support: Georgia Dermatopathology Associates, Atlanta, Georgia, USA. Competing Interests: None

\section{CENTRAL CENTRIFUGAL CICATRICIAL ALOPECIA AMALGAMATED WITH ALOPECIA AREATA: IMMUNOLOGIC FINDINGS}

\author{
Ana Maria Abreu Velez ${ }^{1}$, Bruce R. Smoller ${ }^{2}$, Michael S. Howard ${ }^{1}$ \\ ${ }^{I}$ Georgia Dermatopathology Associates, Atlanta, Georgia, USA \\ ${ }^{2}$ United States and Canadian Academy of Pathology, Augusta, Georgia, USA
}

Corresponding author: Ana Maria Abreu Velez, M.D., Ph.D.

abreuvelez@yahoo.com

\begin{abstract}
Introduction: Both scarring and non-scarring alopecias exist; however, rare cases demonstrate features of both classes.

Case Report: We describe an interesting alopecia case with amalgamated clinical, histologic and immunopathologic features of scarring and non-scarring alopecia. Specifically, the case displays combined features of alopecia areata (AA) and of central centrifugal cicatricial alopecia (CCCA). A 36 year old female presented with symmetric, round, patchy hair loss on her scalp.

Methods: Biopsies for hematoxylin and eosin (H\&E) examination, as well as for special stains, direct immunofluorescence (DIF) and immunohistochemistry (IHC) were performed.

Results: The H\&E biopsy revealed focally diminished hair follicular units, and sebaceous gland damage. Perifollicular concentric fibrosis was confirmed by Verhoeff elastin special staining. Antibodies to micelles were noted. Positive IHC staining for CD4, CD8, CD45 and multiple proteases and protease inhibitors was noted around selected follicular unit remnants.

Conclusion: We present a rare alopecia, combining histologic features of CCCA with additional, selected immunologic features of AA.
\end{abstract}

Key words: Autoimmunity; scarring alopecia; alopecia areata; fibrosing alopecia; CD99

Abbreviations: Alopecia areata (AA), central centrifugal cicatricial alopecia (CCCA), direct immunofluorescence (DIF), immunohistochemistry (IHC), hematoxylin and eosin (H\&E).

\section{Introduction}

Scarring alopecias are often syndromic. Many scarring alopecia immunodermatologic findings are not well studied, especially in overlapping syndromes [1-3]. The most common clinical scarring alopecias include lupus erythematosus, lichen planus, pseudopelade of Brocq, follicular mucinosis, severe folliculitis, dissecting cellulitis, folliculitis decalvans, acne kelodalis nuchae, tinea kerion and favus [1-3]. Overall, immunologic findings derived from direct immunofluorescence (DIF) and/or immunohistochemistry (IHC) in scarring and nonscarring alopecias are not well documented in the literature. Alopecia areata (AA) is classically considered a non-scarring alopecia [4]. Although scarring alopecias may clinically present in a similar manner to AA, discernible differences may also be present. In AA, the hairless patches are usually smaller, more symmetric and round. Scarring alopecias may clinically "burn out"; the hairless spots will then stop increasing in diameter, concomitant with cessation of the clinical etiology [1-3]. Overlap syndromes involving alopecias have been described, but often without significant documentation of DIF and IHC data. Previous attempts to confirm autoantibodies directed against hair follicle components in sera from AA patients have met with difficulty [4-6]. In addition, the majority of perilesional inflammatory cells around hair follicles in AA are either CD4 or CD8 positive lymphocytes.

\section{Case Report}

A 36 year old African American female presented for alterations in her scalp hair. Clinically, she demonstrated symmetric, round patches of alopecia with some burning sensations. Examination also revealed focal non-symmetric, scarring alopecic areas. Skin biopsies for hematoxylin and eosin (H\&E) examination, IHC and multiple fluorochrome DIF were performed. 
Initial processing of the biopsies for H\&E examination, DIF and IHC stains was performed as previously described [57]. Our case was IRB exempt because no patient identifiers were recorded. Skin DIF cryosections were prepared and incubated with multiple fluorochromes as previously reported [7-12]. The H\&E sections demonstrated an unremarkable epidermis with no interface inflammation. Within the papillary and reticular dermis, hair follicular unit density was focally diminished. There were 2-3 hairs per follicular unit, and less than four sebaceous glands per follicular unit (Fig. 1). Sebaceous gland morphology varied from normal to complete destruction. The overall anagen:telogen ratio appeared normal. A mild, superficial, perivascular lymphohistiocytic infiltrate was present. No perifollicular inflammation was seen. Lymphocytes were predominantly present around blood vessels surrounding hair follicular units. Mild perifollicular concentric fibrosis was noted. Diffuse scarring was seen in focal regions of the biopsy, and rare follicular stelae scars were seen. A Verhoeff elastin stain confirmed focal dermal scarring, occupying approximately $25 \%$ of the biopsy area. A Periodic acid Schiff(PAS) stain displayed positive enhancement along basement membrane zone areas of some hair follicular units and eccrine glands (Figs. 1, 2). Finally, an Alcian blue special stain highlighted follicular remnants, with mucin deposition noted within scarring areas (Fig. 2). By IHC, vimentin defined dermal pilosebaceous gland remnants. TIMP1 was positive on remnants of follicular units, and concentrated on sebaceous glands. CD8 positive cells were noted within the perifollicular lymphocytic infiltrate, and also around blood vessels that supplied piloerector muscles and eccrine glands (Fig. 1). Within the isthmus of hair follicles, we also noted CD8 positive staining. CD4 positive cells displayed a similar pattern to the CD8 positive cells. Strong CD45 and CD99 positivity was noted around perifollicular blood vessels. There was also strong positive staining with $\mathrm{IgG}$ and $\mathrm{IgD}$ around hair follicular units; IgD was accentuated around follicular remnants. Positive staining for IgG also was noted in focal areas of the epidermal granular cell layer, within dermal endothelial-mesenchymal cell junctions, and in endothelial cells of perifollicular blood vessels. IgM demonstrated a similar, but stronger staining pattern as IgG. Complement/C $3 \mathrm{c}$ demonstrated a similar pattern to $\mathrm{IgG}$, but with weaker staining within endothelial/mesenchymal dermal cell junctions. By DIF, Complement/C1q showed strong positivity within micelle-like structures near damaged sebaceous glands (Fig. 3). Strongly positive IgD staining was noted within and around hair follicles, and specifically around surrounding blood vessels. Complement/C3c and C1q were also strongly positive within the follicular remnants (Fig. 3 and Tabl. I).
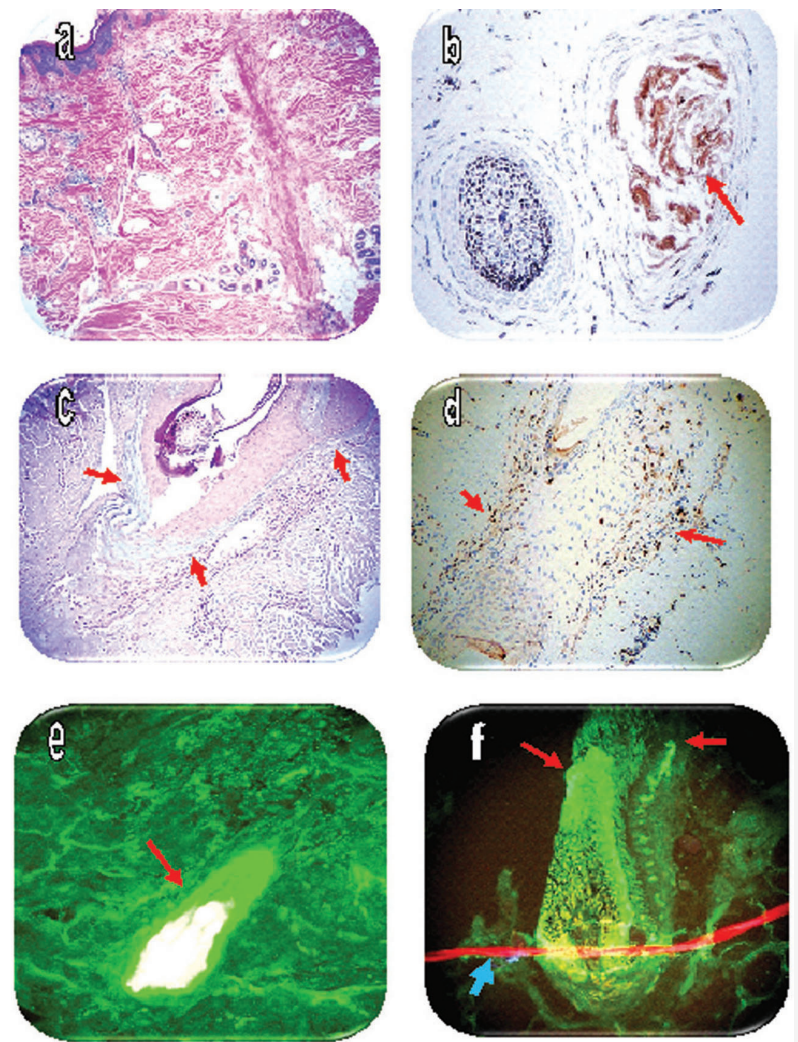

Figure 1. a. A representative H\&E section, demonstrating the decrease in hair follicular units. b. IHC CD8 positive staining inside remnants of a hair follicle (brown staining; red arrow). c. Alcian blue special stain, highlighting reactive mucin deposition in areas of dermal scarring (light blue staining; red arrows). d. IHC CD8 positive staining around a hair follicle, and around adjacent blood vessels(brown staining; red arrows). e. DIF positive staining of a hair bulb remnant, utilizing FITC conjugated anti-human Complement/Clq (yellow/white staining; red arrow). f. DIF positive staining of a hair bulb remnant, utilizing FITC conjugated anti-human IgD; note the staining against several areas within the hair follicle and hair bulb (green staining; red arrows). The thin, horizontal red structure represents a long neurovascular fiber, with its nuclei counterstained with Topro III (red staining; blue arrow). 

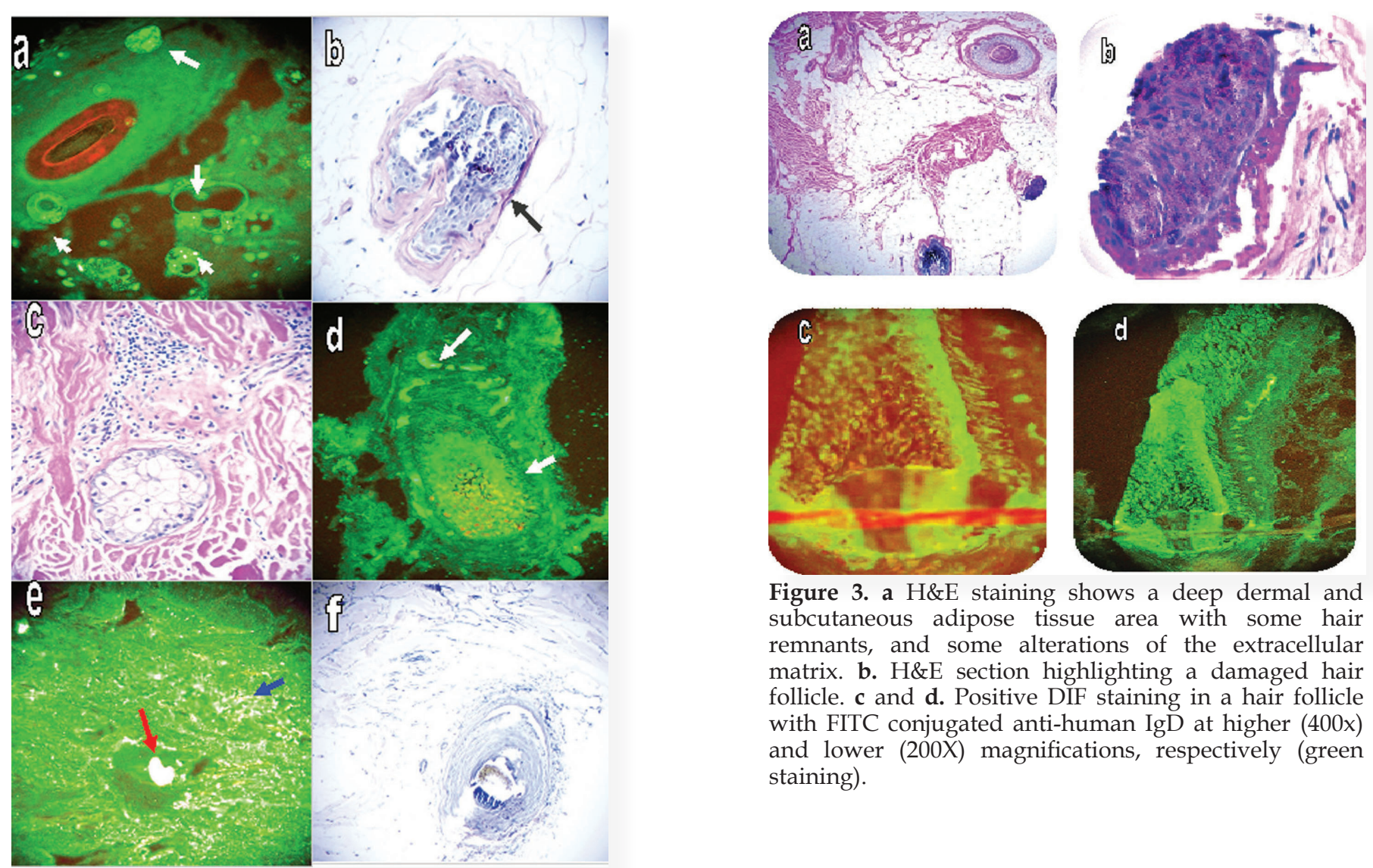

Figure 3. a H\&E staining shows a deep dermal and subcutaneous adipose tissue area with some hair remnants, and some alterations of the extracellular matrix. b. H\&E section highlighting a damaged hair follicle. $\mathrm{c}$ and $\mathrm{d}$. Positive DIF staining in a hair follicle with FITC conjugated anti-human IgD at higher $(400 \mathrm{x})$ and lower (200X) magnifications, respectively (green staining).

Figure 2. a DIF positive staining of a hair follicular unit remnant, utilizing FITC conjugated anti-human IgD; note the micelles formed at the interface of the aqueous and lipid components (round structures; green staining, white arrows). b. Positive PAS special staining along a hair follicle basement membrane (red/purple staining; black arrow). c. H\&E staining, demonstrating sebaceous gland destruction accompanied by inflammation around the remnants of a hair follicular unit; focal scarring is also present. $\mathbf{d}$. Positive DIF staining of a hair bulb remnant and some areas of the hair follicle, utilizing FITC conjugated anti-human Kappa light chains antibody (yellow/green staining; white arrows). e. DIF positive staining with FITC conjugated Complement/C1q antibody of a hair bulb remnant (white staining; red arrow), and several dermal blood vessels (white staining; blue arrow). f. A clinical picture of the alopecia.

\begin{tabular}{|l|l|l|l|}
\hline DIF Antibodies & DIF results & $\begin{array}{l}\text { IHC Antibodies } \\
\text { (all from Dako })\end{array}$ & IHC results \\
\hline IgG-FITC, Dako. & $\begin{array}{l}\text { Positive on hair follicle germinal bulbs } \\
(+++) \text { with additional epidermal } \\
\text { positivity. }\end{array}$ & Vimentin & $\begin{array}{l}\text { Strong reactivity around hair follicular units, } \\
\text { dermal blood vessels and some atrophic eccrine } \\
\text { sweat glands and eccrine ducts. }\end{array}$ \\
\hline IgM-FITC, Dako. & $\begin{array}{l}\text { Positive on hair follicle germinal bulbs, } \\
\text { and one focus outside the sebaceous } \\
\text { glands. }\end{array}$ & LAT & Negative. \\
\hline IgA-FITC, Dako. & Negative. & Metallothionein & Positive in dermal eccrine glands. \\
\hline $\begin{array}{l}\text { Complement/C3- } \\
\text {-FITC, Dako. }\end{array}$ & $\begin{array}{l}\text { Positive on hair follicle germinal bulbs, } \\
\text { and around dermal blood vessels. }\end{array}$ & TIMP-1 & Positive at the bases of a few hair follicles. \\
\hline $\begin{array}{l}\text { Complement/C4- } \\
\text { FITC, Dako. }\end{array}$ & $\begin{array}{l}\text { Positive on hair follicle germinal bulbs, } \\
\text { and in remnants of damaged hair } \\
\text { follicular units. }\end{array}$ & CD99 & $\begin{array}{l}\text { Positive on dermal blood vessels and accentuated } \\
\text { on blood vessels associated with hair follicle } \\
\text { remnants. }\end{array}$ \\
\hline $\begin{array}{l}\text { Fibrinogen-FITC, } \\
\text { Dako. }\end{array}$ & \begin{tabular}{l} 
Positive on hair follicle germinal bulbs. \\
\hline
\end{tabular} & CD8 & $\begin{array}{l}\text { Positive around several dermal blood vessels, and } \\
\text { eccrine ducts close to damaged follicles. }\end{array}$ \\
\hline
\end{tabular}

Table I. Summary of DIF and IHC staining. 


\begin{tabular}{|c|c|c|c|}
\hline DIF Antibodies & DIF results & $\begin{array}{l}\text { IHC Antibodies } \\
\text { (all from Dako) }\end{array}$ & IHC results \\
\hline $\begin{array}{l}\text { Albumin-FITC, } \\
\text { Dako. }\end{array}$ & $\begin{array}{l}\text { Positive staining of the BMZs of the } \\
\text { sebaceous and eccrine glands. Positive } \\
\text { epidermal staining within the corneal } \\
\text { layer, and some pericytoplasmic staining } \\
\text { in keratinocytes of the epidermal } \\
\text { granular cell layer. Positive on hair } \\
\text { follicle germinal bulbs. }\end{array}$ & CD45 & $\begin{array}{l}\text { Positive around dermal blood vessels, close to hair } \\
\text { follicles. }\end{array}$ \\
\hline $\begin{array}{l}\text { IgE -FITC, Goat } \\
\text { anti-human, Vector } \\
\text { Laboratories. }\end{array}$ & Negative. & CD4 & $\begin{array}{l}\text { Positive around dermal blood vessels, close to hair } \\
\text { follicles. }\end{array}$ \\
\hline $\begin{array}{l}\text { Complement/C1q- } \\
\text { FITC, Kent } \\
\text { Laboratories. }\end{array}$ & $\begin{array}{l}\text { Positive on hair follicle germinal bulbs, } \\
\text { in micelles formed after destruction of } \\
\text { sebaceous glands, and in remnants of the } \\
\text { sebaceous glands. }\end{array}$ & Complement/C3c & $\begin{array}{l}\text { Positive around hair follicle germinal bulbs, and } \\
\text { around blood vessels in the dermis. }\end{array}$ \\
\hline $\begin{array}{l}\text { IgD-FITC, Goat } \\
\text { anti-human, Southern } \\
\text { Biotechnology. }\end{array}$ & $\begin{array}{l}\text { Positive on hair follicle germinal bulbs, } \\
\text { with focal additional staining in the } \\
\text { epidermis. Positive at bases of hair } \\
\text { follicle germinal bulbs. }\end{array}$ & Complement/C1q & $\begin{array}{l}\text { Positive around hair follicle germinal bulbs, and } \\
\text { around several dermal blood vessels (especially } \\
\text { those in proximity to hair follicular units). Positive } \\
\text { also on remnants of sebaceous glands. }\end{array}$ \\
\hline $\begin{array}{l}\text { Kappa light } \\
\text { Chains-FITC, Dako. }\end{array}$ & Focal positivity in the hair follicles. & $\operatorname{IgD}$ & Positive in remnants of hair follicular units. \\
\hline $\begin{array}{l}\text { Lambda light } \\
\text { Chains-FITC, Dako. }\end{array}$ & $\begin{array}{l}\text { Pericytoplasmic staining in keratinocytes } \\
\text { of the epidermis. Also positive in hair } \\
\text { follicle germinal bulbs. }\end{array}$ & $\operatorname{IgM}$ & $\begin{array}{l}\text { Positive on hair follicle germinal bulbs, and } \\
\text { around dermal blood vessels. }\end{array}$ \\
\hline
\end{tabular}

Table I. Summary of DIF and IHC staining (continuation).

\section{Discussion}

In clinical practice, often one type of alopecia seems to share selected clinical features with others. Examples include lupus erythematosus (LE) and lichen planus (LP) that may present with an overlap syndrome. Other examples that have been described of possible nosologic overlap include fibrosing alopecia in patterned distributions, and/or Graham-Little syndrome. AA does not classically present with scarring, nor has a definitive DIF pattern been clearly identified for this disease. We present a patient with clinical and immunologic features that resembles an overlap between AA and central centrifugal cicatricial alopecia (CCCA). Moreover, our combined histopathologic and immunologic results did not match any previously described alopecia. Our case demonstrates a scarring alopecia (with positive Alcian blue mucin deposition) and the additional presence of IHC CD4, CD8, CD45 and CD99 cell staining in lesional areas. We also observed the presence of multiple immunoglobulins in these areas, including a very strong response with IgD that seemed to amplify the immune response of the other immunoglobulins and complement. Thus, we document a unique combination of immunoreactants that are not routinely evaluated in routine dermatopathology and dermatoimmunology laboratories. Our results demonstrate a complex pattern of reactivity; some immunoreactivity was noted within follicular germinal bulbs and follicular unit remnants, especially with anti-IgG, $\operatorname{IgM}, \operatorname{IgD}$, complement/C1q, C3c, C4 and fibrinogen.

We also noticed many micelle-like, immunologically reactive structures that were likely formed by the destruction of sebaceous glands and associated mixing of hydrophilic and hydrophobic tissue components. Previous authors have demonstrated similar findings to these in AA [8-10]. According to some researchers, follicular dropout may occur in nonscarring alopecias, leading to a biphasic histologic pattern. The biphasic pattern has been documented in AA, androgenetic alopecia and traction alopecia, and may be pertinent in our case [8-10]. Our DIF and IHC studies also suggest that an antibody and complement-mediated immune response was part of the disease pathogenesis in this patient, along with a T cell response. Overall, we suggest that our case is best categorized as an overlap syndrome. Specifically, our case demonstrates amalgamated pathologic features of non-scarring and scarring alopecias. Consistent with these findings, the patient improved clinically after the application of intralesional corticosteroids. In summary, we describe a case of clinical and histologic CCCA, with additional immunologic features of an AA immune response mediated by antibodies, complement, $\mathrm{T}$ cells and selected proteases. The significance of our findings remains unknown, and warrants further investigation.

\section{REFERENCES}

1. Templeton SF, Solomon AR. Scarring alopecia: a classification based on microscopic criteria. J Cutan Pathol. 1994;21:97-109.

2. 2. Inchara YK, Tirumalae R, Kavdia R, Antony M. Histopathology of scarring alopecia in Indian patients. Am J Dermatopathol. 2011;33:461-7. 
3. Jautová J, Jarcusková D, Ficová M, Dubivská M. Alopecia areataan autoimmune disorder? Bratisl Lek Listy. 1995;96:144-7.

4. Tobin DJ, Bystryn JC. Immunity to hair follicles in alopecia areata. J Invest Dermatol. 1995;(Suppl):13S-14S.

5. Abreu Velez AM, Howard MS, Loebl AM: Autoreactivity to sweat and sebaceous glands and skin homing T cells in lupus profundus. Clin Immunol. 2009;132:420-4.

6. Abreu Velez AM, Girard JG, Howard MS: Antigen presenting cells in a patient with hair loss of and systemic lupus erythematosus. North Am J Med Sci. 2009;1:205-10.

7. Abreu Velez AM, Klein AD, Howard MS. Survivin, p53, MAC, Complement/C3, fibrinogen and HLA-ABC within hair follicles in central and centrifugal cicatricial alopecia. N Am J Med Sci. 2011;3:292-5.
8. Tobin DJ, Hann SK, Song MS, Bystryn JC. Hair follicle structures targeted by antibodies in patients with alopecia areata. Arch Dermatol. 1997;133:57-61.

9. Muller HK, Rook AJ, Kubba R. Immunohistology and autoantibody studies in alopecia areata. Br J Dermatol. 1980;102:609-10.

10. Bystryn JC, Orentreich N, Stengel F. Direct immunofluorescence studies in alopecia areata and male pattern alopecia. J Invest Dermatol. 1979;73:317-20.

Copyright by Ana Maria Abreu Velez, et al. This is an open access article distributed under the terms of the Creative Commons Attribution License, which permits unrestricted use, distribution, and reproduction in any medium, provided the original author and source are credited. 